OPEN ACCESS

Edited by:

Wei Wang,

Henan Agricultural University, China

Reviewed by:

Alejandro Pereira-Santana, CONACYT Centro de Investigación y Asistencia en Tecnología y Diseño del Estado de Jalisco (CIATEJ), Mexico lan S. Wallace,

University of Nevada, Reno, United States

*Correspondence:

Yi-Sheng Cheng

chengys@ntu.edu.tw

tThese authors have contributed equally to this work and share first authorship

Specialty section: This article was submitted to Plant Proteomics and Protein

Structural Biology, a section of the journal Frontiers in Plant Science

Received: 25 February 2021 Accepted: 05 May 2021

Published: 31 May 2021

Citation:

Hsin K-T, Yang T-J, Lee Y-H and Cheng Y-S (2021) Phylogenetic and Structural Analysis of NIN-Like Proteins With a Type I/II PB1 Domain

That Regulates Oligomerization for Nitrate Response.

Front. Plant Sci. 12:672035. doi: 10.3389/fpls.2021.672035

\section{Phylogenetic and Structural Analysis of NIN-Like Proteins With a Type I/II PB1 Domain That Regulates Oligomerization for Nitrate Response}

\author{
Kuan-Ting Hsin ${ }^{1+}$, Tzu-Jing Yang ${ }^{2,3,4 t}$, Yu-Hsuan Lee ${ }^{1}$ and Yi-Sheng Cheng ${ }^{1,4,5 *}$
}

\begin{abstract}
' Department of Life Science, College of Life Science, National Taiwan University, Taipei, Taiwan, ${ }^{2}$ Institute of Biological Chemistry, Academia Sinica, Taipei, Taiwan, ${ }^{3}$ Institute of Biochemical Sciences, College of Life Science, National Taiwan University, Taipei, Taiwan, ${ }^{4}$ Institute of Plant Biology, College of Life Science, National Taiwan University, Taipei, Taiwan, ${ }^{5}$ Genome and Systems Biology Degree Program, College of Life Science, National Taiwan University, Taipei, Taiwan
\end{abstract}

Absorption of macronutrients such as nitrogen is a critical process for land plants. There is little information available on the correlation between the root evolution of land plants and the protein regulation of nitrogen absorption and responses. NIN-like protein $(N L P)$ transcription factors contain a Phox and Bem1 (PB1) domain, which may regulate nitrate-response genes and seem to be involved in the adaptation to growing on land in terms of plant root development. In this report, we reveal the NLP phylogeny in land plants and the origin of NLP genes that may be involved in the nitrate-signaling pathway. Our NLP phylogeny showed that duplication of NLP genes occurred before divergence of chlorophyte and land plants. Duplicated NLP genes may lost in most chlorophyte lineages. The NLP genes of bryophytes were initially monophyletic, but this was followed by divergence of lycophyte NLP genes and then angiosperm NLP genes. Among those identified NLP genes, PB1, a protein-protein interaction domain was identified across our phylogeny. To understand how protein-protein interaction mediate via PB1 domain, we examined the PB1 domain of Arabidopsis thaliana NLP7 (AtNLP7) in terms of its molecular oligomerization and function as representative. Based on the structure of the PB1 domain, determined using small-angle $x$-ray scattering (SAXS) and site-directed mutagenesis, we found that the NLP7 PB1 protein forms oligomers and that several key residues (K867 and D909/D911/E913/D922 in the OPCA motif) play a pivotal role in the oligomerization of NLP7 proteins. The fact that these residues are all conserved across land plant lineages means that this oligomerization may have evolved after the common ancestor of extant land plants colonized the land. It would then have rapidly become established across land-plant lineages in order to mediate protein-protein interactions in the nitrate-signaling pathway.

Keywords: NIN-like protein, PB1, duplication, monophyly, protein-protein interaction, land plant 


\section{INTRODUCTION}

The evolution of land plants involved a series of adaptations to living in diverse terrestrial environments, including the evolution of roots-a key organ that allows plants to absorb macronutrients. Among macronutrients, nitrogen plays an important role in plant growth and development, and nitrates are the most abundant inorganic form of $\mathrm{N}$ in soils (Marchive et al., 2013; Konishi and Yanagisawa, 2014, 2019; Liu et al., 2017). Nitrates are known to be involved in plant growth and development, and to serve as signaling molecules that are involved in the primary nitrate response (PNR) (Gowri et al., 1992; Liu et al., 2017). For example, the relative growth rate of Lactuca sativa seedlings was 10 times higher in a nitrate-treated control than that of seedlings in a zero-nitrate treatment (Walker et al., 2001), suggesting the important role of nitrates in plant development. The PNR triggers hundreds of genes at the transcription level in response to nitrate concentration changes in the environment (Gowri et al., 1992). Molecular evidence reveals that NIN-like protein (NLP) transcription factors (TFs) are key players in the PNR. For example, $\beta$-glucuronidase (GUS) expression in an NLP6 transgenic line of Arabidopsis can be observed after nitrate treatment, whereas no GUS signal is observed after $\mathrm{KCl}$ treatment (Konishi and Yanagisawa, 2013). This suggests that NLP may serve as a nitrate-response TF.

Nine NLPs (AtNLP1-AtNLP9) have so far been identified in Arabidopsis, and they have $34.3-72.3 \%$ similarity in their aminoacid sequences. Because of this high level of similarity, it has been proposed that there is functional redundancy within the nine AtNLPs, which code for nitrate-inducible gene expression (Konishi and Yanagisawa, 2019). Recently, phylogeny analysis of NLPs in plants has shown that these nine AtNLPs can be categorized into three groups, implying some functional divergence (Schauser et al., 2005; Mu and Luo, 2019; Liu and Bisseling, 2020). Group 1 comprises AtNLP1-5 and may be involved in nodule formation. This hypothesis is supported by data on the protein NODULE INCEPTION (NIN) in Medicago truncatula (Liu et al., 2019). Group 2 comprises AtNLP8 and 9, and in Arabidopsis, AtNLP8 has been found to be associated with nitrate-promoted seed germination (Yan et al., 2016). Group 3 comprises AtNLP6 and -7, and Arabidopsis thaliana NLP7 (AtNLP7) is known to be involved in regulating the expression levels of nitrate-inducible genes (Castaings et al., 2009; Wang et al., 2009). Therefore, these three groups of NLPs have functionally diverged over the course of evolution, and they now play roles in either nodule formation, nitrate-signaling response, or nitrate-induced gene expression.

Based on the amino-acid sequence alignment of NLPs, three conserved regions have been identified: the nitrate-response domain (NRD), the RWP-RK domain, and the Phox and Bem1 (PB1) domain (Konishi and Yanagisawa, 2014, 2019). The NRD domain is known to mediate the activation of NLPs via a conserved serine residue (serine 205 in AtNLP7) when nitrate is provided (Yan et al., 2016; Liu et al., 2017). The RWP-RK motif is named for the conserved amino-acid sequence Arg-Trp-Pro-XArg-Lys (where $\mathrm{X}$ indicates any amino acid), which can bind to nitrate-responsive cis-elements (NREs) located in the promoter region of nitrate-inducible genes (like NIR1) in Arabidopsis (Konishi and Yanagisawa, 2010; Chardin et al., 2014; Sato et al., 2016; Mu and Luo, 2019). The PB1 domain may contain either a type I or a type II domain, or both (Mu and Luo, 2019). The type I domain comprises beta I, beta II, and alpha I, which contains a conserved lysine residue. The type II domain comprises beta III, beta IV, alpha II, and beta V. AtNLPs contain both type I and type II domains (type I/II PB1 domain), which can interact with NLPs that contain a type I, type II, or type I/II domain (Sumimoto et al., 2007). Interaction between two PB1 domains is mediated by four core amino-acid residues (K867, D909, D911, and E913), which may facilitate NLP-NLP homodimerization (Konishi and Yanagisawa, 2019). The homodimerization of NLPs is necessary for fully promoting nitrate-induced gene expression in the presence of nitrate (Konishi and Yanagisawa, 2019). Proteinprotein interaction and quantitative polymerase chain reaction (qPCR) experiments reveal that the PB1 domain plays a key role in regulating nitrate-inducible gene expression. However, how NLP proteins interact via the PB1 domain remains unclear.

In this report, the order of divergence of $N L P$ genes across land plants was reconstructed to investigate how the evolution of NLPs correlates with root development in land plants. The NLPS of bryophytes were used as the root to infer the order of NLP gene divergence in angiosperms. In addition, the oligomerization state and protein structure of the AtNLP7 PB1 domain were revealed by size-exclusion chromatography (SEC) and small-angle X-ray scattering (SAXS). The structure and protein-protein interaction of the PB1 domain were built and confirmed using homology modeling and site-directed mutagenesis. Combining our findings regarding the evolutionary path and the molecular structure and function of AtNLP7 in plants, we conclude that the functional divergence of NLPs may be correlated with the development of plant roots in terms of nitrate response and specialized nodule formation, which occur via similar types of molecular regulation.

\section{MATERIALS AND METHODS}

\section{NLP Phylogeny Reconstruction}

First, the core amino-acid residues of the PB1 domain in green plants were labeled in Bioedit to identify conserved NLP genes (Hall, 1999). Viridiplantae is composed of green algae and land plants. In reconstructed species phylogeny, seven lineages are identified within Viridiplantae, including chlorophyte, charophytes, bryophyte, lycophyte, monilophyte, gymnosperm and angiosperm (Finet et al., 2010). We aimed to reconstruct evolutionary history of NLP genes across major lineages within Viridiplantae. To achieve this goal, NLP genes of major Viridiplantae lineages were obtained from the online Phytozome database ${ }^{1}$ (Goodstein et al., 2012), PLAZA gymnosperm ${ }^{2}$ and the database of the National Center for Biotechnology Information (NCBI). The NLP genes of A. thaliana (AtNLP1 to AtNLP9) were used as templates for identifying the NLP genes of other species. Species selected

\footnotetext{
${ }^{1}$ https://phytozome.jgi.doe.gov/pz/portal.html\#

${ }^{2}$ https://bioinformatics.psb.ugent.be/plaza/versions/gymno-plaza/
} 
for this purpose included Micromonas pusilla, Micromonas sp., Marchantia polymorpha, Selaginella moellendorffi, Amborella trichopoda, gymnosperm, basal angiosperms, monocots, and eudicots (for details see Supplementary Table 1). To conduct a comprehensive search for the NLP genes of the selected species, the default threshold value and tblastx were set for each search. Only sequences containing an RWP-RK domain and a PB1 domain were used for phylogeny reconstruction.

Second, all NLP sequences were aligned using the MUSCLE algorithm (Edgar, 2004) in MEGA v.6 (Tamura et al., 2013). Next, the resulting alignment matrix was visually refined based on amino-acid translations using Bioedit (Hall, 1999). The HKY85 + G + I model was selected as the best-fitting model based on the Bayesian information criterion (BIC) (Schwarz, 1978). Both Bayesian inference (BI) and maximum likelihood (ML) methods were used to infer the relationships between the NLP genes, using the PhyML 3.0 online interface (Guindon et al., 2010). The HKY85 + G + I model derived from jModelTest2 (Darriba et al., 2012) was applied. Statistical support for the nodes was assessed using an approximate likelihood ratio test (aLRT) (Anisimova and Gascuel, 2006) and a Bayesian-like transformation of the aLRT (aBayes) (Anisimova et al., 2011) for the ML and BI findings, respectively. The conventional bootstrap algorithm was conducted with 1000 replicates. To present the gene relationships clearly, we present the cladogram of the landplant NLP genes in the main article, but the phylogram of the NLP genes is included as Supplementary Figure 1.

\section{AtNLP7 PB1 Domain Construction}

We first attempted to express AtNLP7 protein. However, the full-length AtNLP7 protein tended to form an aggregate and precipitate in our pre-test. Instead, we focused on expressing PB1 domain, which serving as protein-protein interaction domain on AtNLP7. For protein expression and purification, the wild type AtNLP7 PB1 domain was PCR-amplified from the plasmid pDL2Nx-NLP7 PB1, kindly provided by Dr. Yi-Fang Tsay (Institute of Molecular Biology, Academia Sinica), using the PB1-EcoRI primer (5'-ATTCCGAATTCCCAAAGGAA GAGGCCATTGC-3') and the PB1-HindIII primer (5'-AATT AAGCTTCTAGCAGGAGCTCCCTAGATTTGTCG-3'). It was then subcloned into the expression vector pET28a such that the recombinant protein contained a six-histidine tag and thrombin cleavage site sequences at the $\mathrm{N}$-terminus. Site-directed mutagenesis was performed using the QuikChange Lightning Site-Directed Mutagenesis Kit (Agilent, Santa Clara, CA, United States) to create NLP7 PB1m1 (K867A), NLP7 PB1m2 (D909A/D911A), and NLP7 PB1m3 (K867A/D909A/D911A), using specific primers (Supplementary Table 2).

\section{Protein Expression and Purification}

The wild type and NLP7 PB1 mutants were expressed using Escherichia coli strain BL21 (DE3). Cultures were incubated at $37^{\circ} \mathrm{C}$ to an $\mathrm{OD}_{600}$ of $0.4-0.6$ and induced using $0.1 \mathrm{mM}$ isopropyl $\beta$-D-1-thiogalactopyranoside (IPTG), then grown for $8 \mathrm{~h}$ at $37^{\circ} \mathrm{C}$. Bacterial cells were pelleted and lysed by sonication in $100 \mathrm{~mL}$ of buffer A (20 mM Tris- $\mathrm{HCl}$ pH 8.8, $0.5 \mathrm{M} \mathrm{NaCl}, 10 \%$ [v/v] glycerol) for wild type NLP7 PB1 (NLP7 PB1wt) and buffer B (20 mM NaH $\left.2 \mathrm{PO}_{4}, \mathrm{pH} 7.4,0.5 \mathrm{M} \mathrm{NaCl}\right)$ for the mutants.
After sonication, the cell debris were centrifuged for $25 \mathrm{~min}$ at $12,500 \times g$. The supernatants were filtered through a $0.45 \mu \mathrm{m}$ filter (Sartorius) and loaded into a $5 \mathrm{~mL} \mathrm{Ni}^{2+}$-Sepharose resin column (HisTrap FF, GE Healthcare). The column was washed with 10 times the column volume of buffer A for NLP7 PB1wt or buffer B for the mutants, complemented with $40 \mathrm{mM}$ imidazole. The target proteins were bound and eluted with buffer A for NLP7 PB1wt or buffer B for the mutants, complemented with $500 \mathrm{mM}$ imidazole. The molecular mass of the purified 6xHisNLP7 PB1 domain was 15.6 kDa (Supplementary Figures 2-4).

\section{Size-Exclusion Chromatography}

For advanced purification, all proteins were passed through a Superdex S-75 column (GE Healthcare) with buffer A. The molecular mass of all four proteins was estimated in buffer A using a Superdex S-75 column and calibrated with gel filtration standard markers [Bio-Rad: $\gamma$-globulin (bovine), $158 \mathrm{kDa}$; ovalbumin (chicken), $44 \mathrm{kDa}$; myoglobin (horse), $17 \mathrm{kDa}$; and vitamin B12, $1.35 \mathrm{kDa}$. The molecular mass of NLP7 PB1 was also determined using a Superdex S-75 column and calibrated with gel filtration standard markers in buffer C $[20 \mathrm{mM}$ Tris, $\mathrm{pH} 8.8,0.5 \mathrm{M} \mathrm{NaCl}, 10 \%$ glycerol, $5 \mathrm{mM}$ reduced glutathione (GSH), $1 \mathrm{mM}$ tris(2-carboxyethyl)phosphine (TCEP)]. Buffer $\mathrm{C}$ was designed to reduce protein aggregation and increase the solubility of the protein samples. After SEC, all proteins were concentrated using Amicon Ultra-0.5 centrifugal filters (Merck, Darmstadt, Germany) and quantified using a DS-11 spectrophotometer (DeNovix).

\section{Small-Angle X-Ray Scattering}

Small-angle $\mathrm{x}$-ray scattering was performed at the beamline (BL23A1), National Synchrotron Radiation Research Center (NSRRC), Hsinchu, Taiwan. Protein samples were placed in a $3 \mathrm{~mm}$ four-loading rocking cell with Kapton and collected using three different concentrations of NLP7 PB1wt dimer: 0.9, 1.2, and $1.5 \mathrm{mg} / \mathrm{mL}$. The order of sample loading for data collection was from lowest concentration to highest. The experimental parameters for SAXS were as follows: photon energy, $15 \mathrm{keV}$; sample thickness, $2.641 \mathrm{~mm}$; distance-to-sample, $4 \mathrm{~m}$. For the NLP7 PB1 domain, the scattering vector $(q)$ ranged from 0.007 to $0.35 \AA^{-1}$, where $q=4 \pi \sin \theta / \lambda$. Data collection was performed after a brief delay of $5 \mathrm{~s}$, for the transmission to normalize, for a continuous period of $200 \mathrm{~s}$ of $\mathrm{x}$-ray exposure.

The SAXS data were analyzed using PRIMUS (Konarev et al., 2003) to estimate the state of each protein sample in the buffer condition, and model building and three-dimensional (3D) surface reconstruction was conducted using GNOM (Svergun, 1992), DAMMIF (Franke and Svergun, 2009), and Situs 2.7.3 (Wriggers and Chacón, 2001). The homology model of the NLP7 PB1 dimer was built using Modeller 9.15 (Eswar et al., 2006), based on the template of the PKC-p62 complex [Protein Data Bank (PDB) ID: 4MJS], and fitted into a volume map using UCSF Chimera (Pettersen et al., 2004).

\section{Dynamic Light Scattering}

The protein samples were subjected to dynamic light scattering (DLS) after advanced purification by SEC. All the mutants were stored in buffer A. However, NLP7 PB1wt was analyzed 
in both buffer A and buffer C. Data were collected using a Zetasizer Nano ZS DLS instrument (Malvern Instruments, Malvern, United Kingdom) equipped with $50 \mathrm{~mW}$ laser fiber. An appropriate refractive index, viscosity (10\% glycerol), and temperature $\left(25^{\circ} \mathrm{C}\right)$ was set for each sample.

\section{RESULTS}

\section{Divergence Order of NLP Genes Is in Accordance With the Evolutionary Trend of Land Plants}

To obtain an overview of NLP evolution history across Viridiplantae, we first accessed the available databases to retrieve NLP homologs from representative species, like $M$. pusilla and M. polymorpha (see Supplementary Table 1 for details). In total, 61 NLP homologs containing the RWP-RK and PB1 domains were obtained for the 17 selected species. At the N-terminal, a conserved core amino-acid residue, lysine $(\mathrm{K})$, was observed across all the NLP proteins we used (Figure 1, red arrow). In addition, three conserved core residues within the OPCA motif at the C-terminal were identified (Figure 1). In M. polymorpha, only one NLP gene could be identified. In contrast, multiple $N L P S$ were identified in the other non-vascular and vascular plants we selected.

To infer the divergence process of land-plant NLP genes, we reconstructed the evolutionary history of NLP genes using both BI and ML algorithms. Only nodes with BI or ML probability values over 0.8 were regarded as reliable clades. According to our criteria, three major clades can be inferred across the NLP phylogeny (Figure 2). The first clade appeared at the base of the phylogeny and comprised bryophytes (Figure 2, BI: -, aLRT: 0.91). Within the first clade, the NLP homolog found in $M$. polymorpha (Mapoly0083s0040) represented the basal lineage, following the divergence of the NLP genes of Physcomitrella patens and S. moellendorffii. In the bryophyte clade, the NLP homologs in $P$. patens and $S$. moellendorffi each formed monophyletic groups. The second clade comprised lycophyte NLP genes. The third clade comprised the NLP gene homologs found in gymnosperm and angiosperms. The divergence of the gymnosperm and angiosperm NLP gene homologs may have occurred in the common ancestor of seed plant, since gymnosperm, basal angiosperms, monocots, and most dicots have NLP genes representing all three NLP groups (Figure 2, divergence II, BI: 1, aLRT: 1). Within the seed plant clade, three distinct clades were identified: Group 1, Group 2, and Group 3. The Group 2 NLP genes diverged first (Figure 2, BI: 1, aLRT: 1), followed by the divergence between Group 3 and Group 1 NLP genes (Figure 2, divergence III, BI: 0.98, aLRT: 1). In Group 2, the NLP homolog that diverged first was that of Pseudotsuga menziesii PME25376, which was followed by the divergence of the NLP homolog in basal angiosperm, monocots, then the divergence of that in the basal dicot Aquilegia coerulea Aqcoe3G052100, and finally the divergence of the NLP homologs in the other dicots. This order of divergence (basal angiosperms, then monocots, then the basal dicot, and finally the other dicots), was also seen in Groups 1 and 3 (NLP gene of gymnosperm may lost in Group 3). Our phylogeny shows that the order of divergence of the NLP genes is in accordance with the species divergence process that has occurred since plants colonized the land.

\section{The AtNLP7 PB1 Domain Exhibits a Concentration-Dependent Oligomerization State in vitro}

We first attempted to resolve the protein structure of AtNLP7. However, the full-length AtNLP7 protein tended to form an aggregate and precipitate in our pre-test. Therefore, we tried to determine the structure of the PB1 domain, which is the proteinprotein interaction domain, instead of the full protein structure of AtNLP7. During the protein purification of the AtNLP7 PB1 domain, we observed that the target $\mathrm{PB} 1$ protein tended to enter a dimer or oligomer state in vitro after SEC (Figure 3A). To examine whether the concentration of the protein solution affected the oligomerization state of the target protein, we diluted the AtNLP7 PB1 protein solution to 5, 3, 2, or $1 \mathrm{mg} / \mathrm{mL}$. The DLS results showed that as the protein concentration increased, the molecular mass of the AtNLP7 PB1 protein increased: it was $198,107,85$, and $41 \mathrm{kDa}$ for the four concentrations, respectively (Figures 3B-E). Our SEC results thus show that the oligomerization state of AtNLP7 PB1 is affected by the concentration of its solution.

\section{Small-Angle X-Ray Scattering Confirmed That the Lowest Concentration of the NLP7PB1 Domain Formed a Homodimer}

The SAXS technique has been widely used to monitor molecular size, shape, and aggregation state (Kikhney and Svergun, 2015). Our previous results had suggested that the AtNLP7 PB1 domain protein formed a homodimer at the lowest protein solution concentration $(1 \mathrm{mg} / \mathrm{mL})$. To obtain a comprehensive understanding of the AtNLP7 PB1 domain protein, the protein solution was diluted to $0.9,1.2$, and $1.5 \mathrm{mg} / \mathrm{mL}$ for SAXS data collection (Figure 4A). The SAXS data processing in Primus showed that the NLP7 PB1 domain tended to aggregate when its protein concentration reached $1.5 \mathrm{mg} / \mathrm{mL}$. These results were consistent with those from the DLS analysis (Figure 1B-E). Furthermore, the distance distribution function for NLP7 PB1 (the P(r) plot in GNOM) adopted a dumbbelllike shape (Figure $\mathbf{4 B}$ ). The results could be used to estimate an average radius of gyration $\left(\mathrm{R}_{g}\right)$ of $25.45 \AA$ and a maximum particle size $\left(\mathrm{D}_{\max }\right)$ of $71.14 \AA$ (Figure $4 \mathrm{C}$ ) for the protein. Based on the DAMMIF calculation, a dummy atom model was generated using $a b$ initio modeling (Figure 4D) and a low-resolution $3 \mathrm{D}$ structural envelope was reconstructed using Situs 2.7.3. The structural envelope of NLP7 PB1 had a dumbbell shape. We also built a structural model for NLP7 PB1 by homology modeling using the template of the PKCp62 complex (PDB ID: 4MJS), a PB1-PB1 domain interaction. A dimeric form of NLP7 PB1 was generated by homology modeling and superimposed onto the $3 \mathrm{D}$ structural envelope from the SAXS analysis using Chimera. The superimposition 


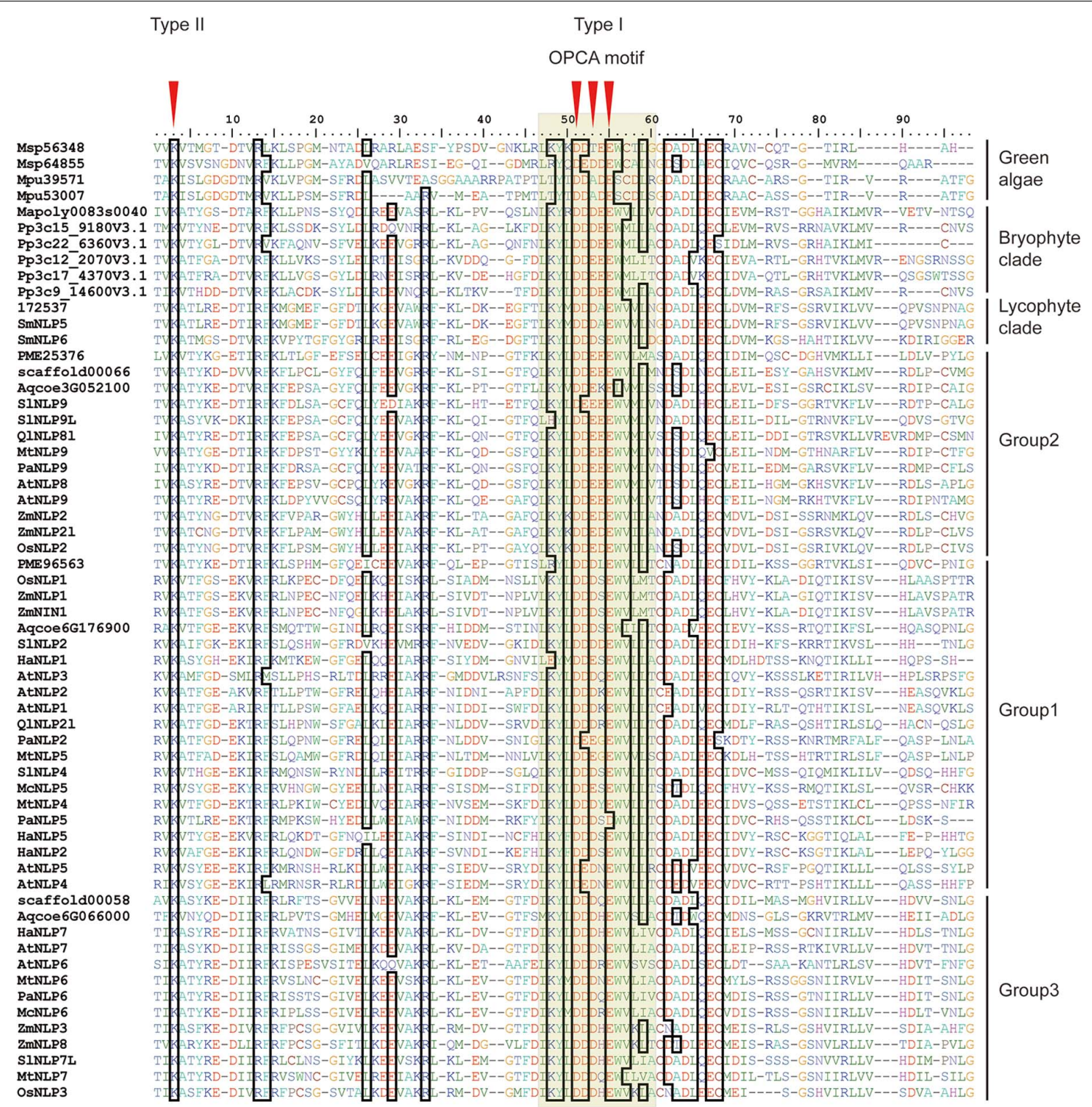

FIGURE 1 | Alignment of land plant PB1 domains. First red arrow indicates core amino-acid residues in the type II motif; Second, third, and fourth arrows indicate core amino-acid residues in the type I motif. The gray hollow box indicates the OPCA motif. Sequence codes are identical to those in Figure $\mathbf{2}$.

result indicated that NLP7 PB1 should exist as a homodimer in vitro (Figure $4 \mathrm{E}$ ).

\section{The AtNLP7 PB1 Domain Dimer Is Formed by Salt-Bridge Interactions}

Based on the model of the AtNLP7 PB1 dimer, we used the PDBePISA server (Krissinel and Henrick, 2007) to analyze the interface and reveal possible interactions (Figure 5A). The results showed that the interface area of the NLP7 PB1 dimer model was approximately $438 \AA^{2}$ (Figure 5B) and that the dimerization of NLP7 PB1 occurs via salt-bridge interactions between K867 (a positively charged residue) and the OPCA motif (negatively charged residues: D909/D911/E913/D922)
(Figure 5C). In addition, there are several extra hydrogen bonds and salt-bridge interactions between Phe877 and Ser881 in the positively charged interface and Glu925 and Leu916 in the negatively charged interface. These may contribute to the dimerization of the NLP7 PB1 domain via weak interactions.

\section{Interactions Between the K867 and OPCA Motifs Play Pivotal Roles in Oligomerization of the NLP7 PB1 Domain}

Based on the 3D structure envelope and homology model (Figures $4 \mathrm{E}, 5 \mathrm{~A}, \mathrm{C}$ ), we hypothesized that the dimerization of NLP7 PB1 should depend on the interaction between the K867 residue (type II motif) and OPCA (type I motif). 


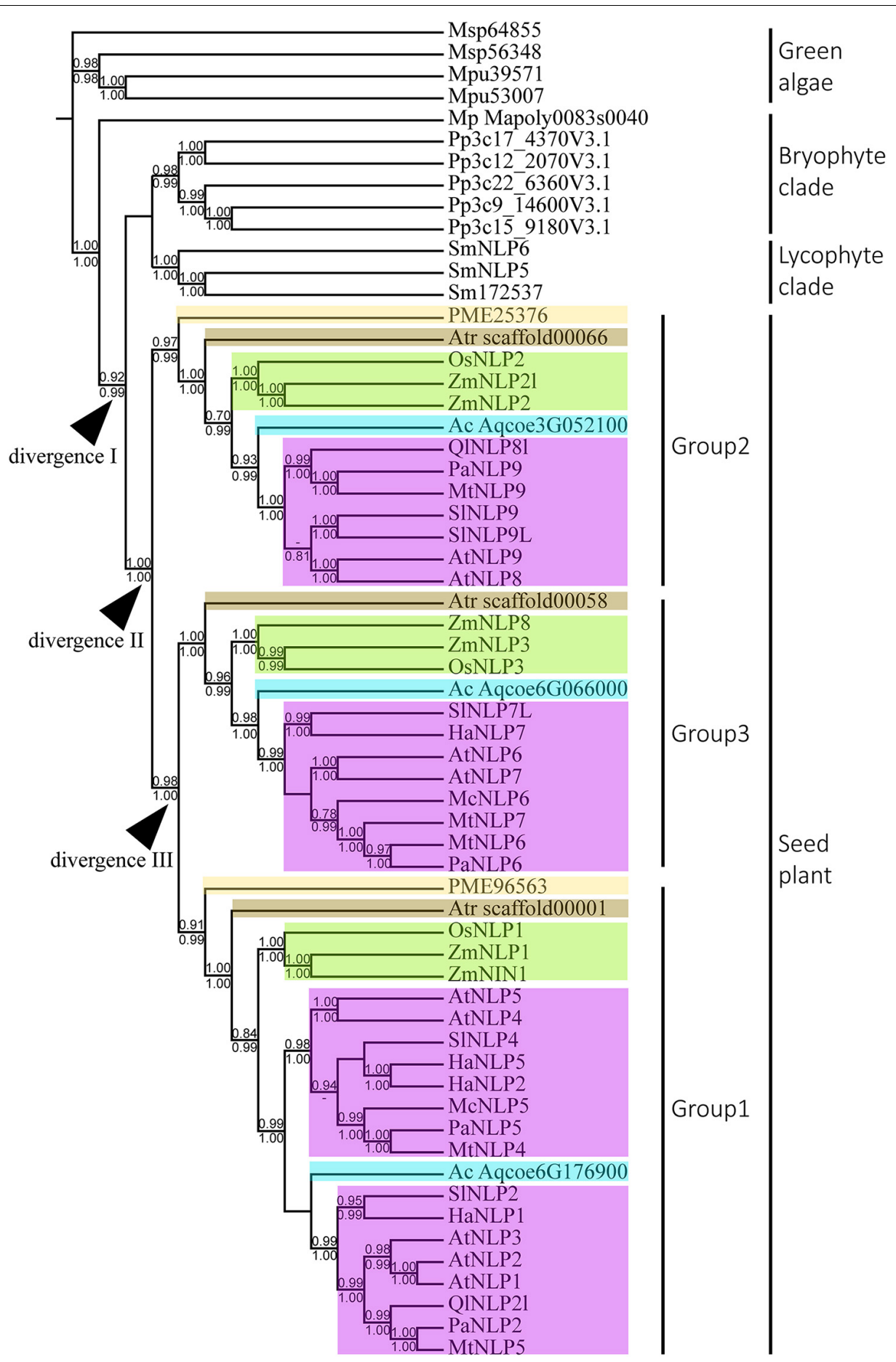

FIGURE 2 | Phylogeny and divergence events of NIN-like proteins (NLPS) in land plants. The cladogram was derived from a maximum likelihood (ML) analysis and shows average branch lengths. Numbers along the branches are Bayesian inference (BI) and ML approximate likelihood values (aLRT). (-) indicates a branch-support value lower than 0.7 in either the BI or the aLRT algorithm. NLP genes of specific plant clades are colored: Amborella trichopoda (brown), Oryza and Zea mays (green), Aquilegia coerulea (blue), gymnosperm (light yellow), and angiosperms (purple). Divergences I to III indicate inferred divergence events. Mp denotes Marchantia polymorpha; Pp denotes Physcomitrella patens; Sm denotes Selaginella moellendorffii; Atr denotes Amborella trichopoda; PME denotes Pseudotsuga menziesii; Os denotes Oryza sativa; Zm denotes Zea mays; Ac denotes Aquilegia coerulea; QI denotes Quercus lobata; Pa denotes Parasponia andersonii; Mt denotes Medicago truncatula; At denotes Arabidopsis thaliana; SI denotes Solanum lycopersicum; Ha denotes Helianthus annuus; Mc denotes Momordica charantia. 


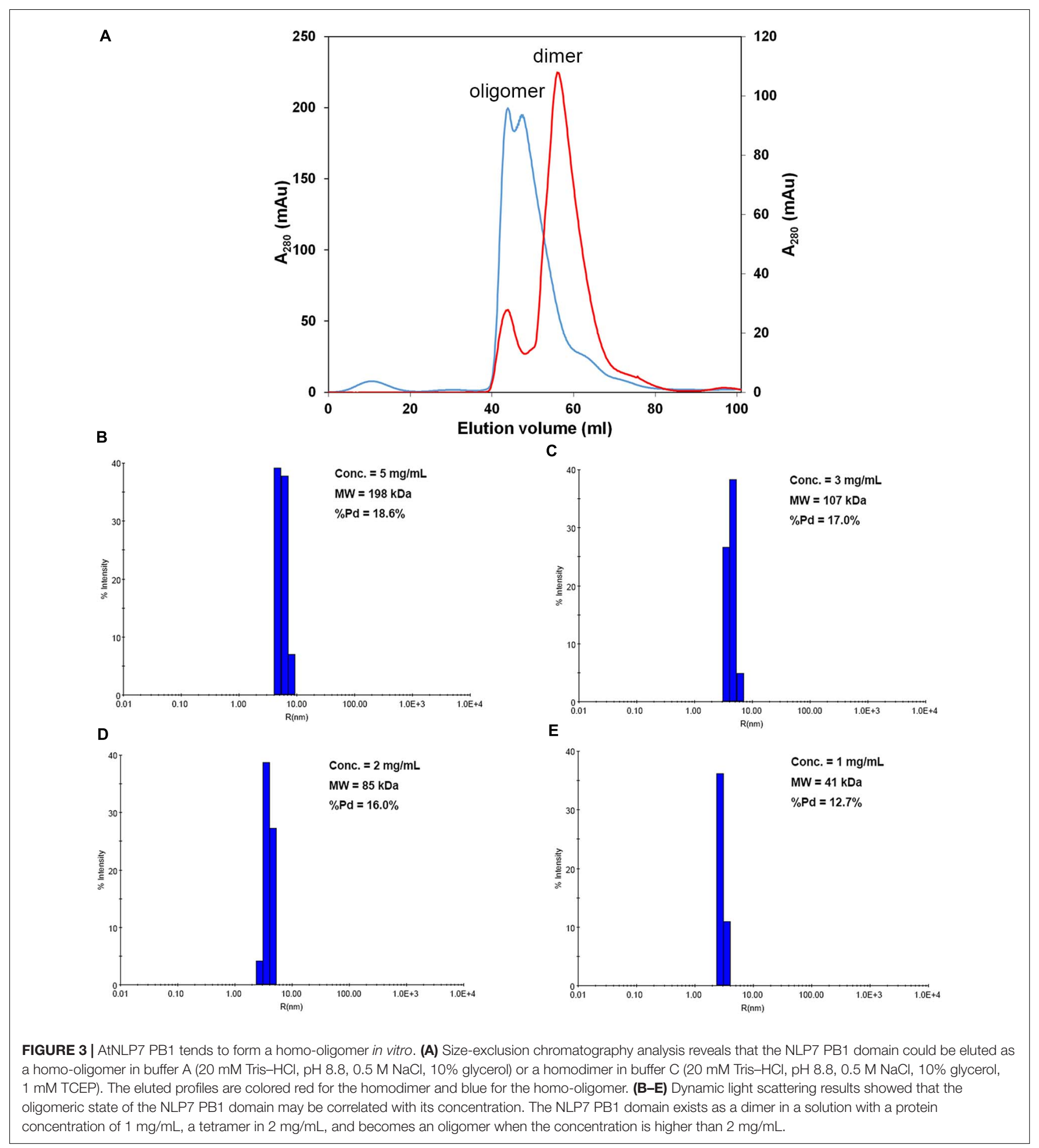

Hence, we mutated the putative interactive residues $(\mathrm{PB} 1 \mathrm{~m} 1$, K867A; PB1m2, D909A/D911A; PB1m3, K867A/D909A/D911A) to disrupt the dimerization or oligomerization of NLP7 PB1 (Figure 6D). Indeed, the SEC results showed that all the mutants were eluted as monomers (Figures 6A,B). We thus surmised that the $\mathrm{K} 867$ residue plays a major interacting role in the positively charged interface to maintain the oligomerization/dimerization. Additionally, we utilized DLS to estimate the molecular weight and homogeneity of all protein samples. These were $81 \mathrm{kDa}$ for NLP7 PB1wt, $27 \mathrm{kDa}$ for NLP7 PB1m1, $27 \mathrm{kDa}$ for NLP7 PB1m2, and $28 \mathrm{kDa}$ for NLP7 PB1m3. Also, all the samples had polydispersity values lower than $20 \%$, indicating that they 
A

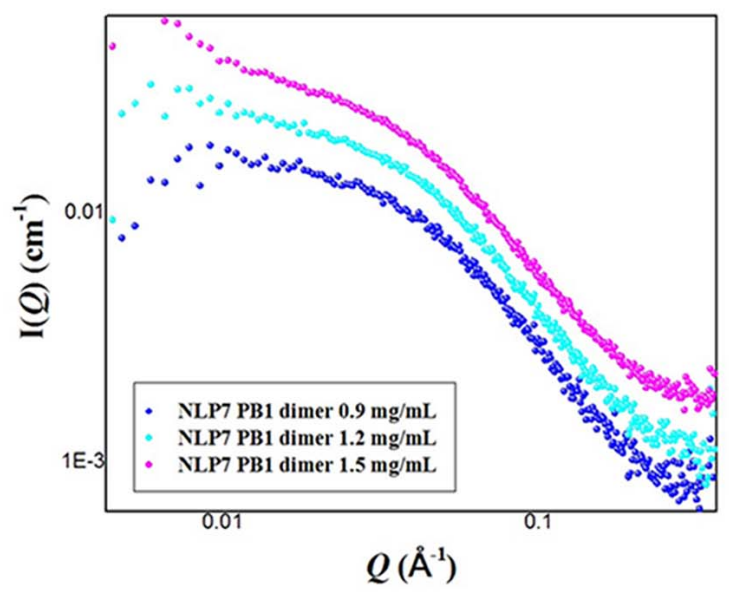

B

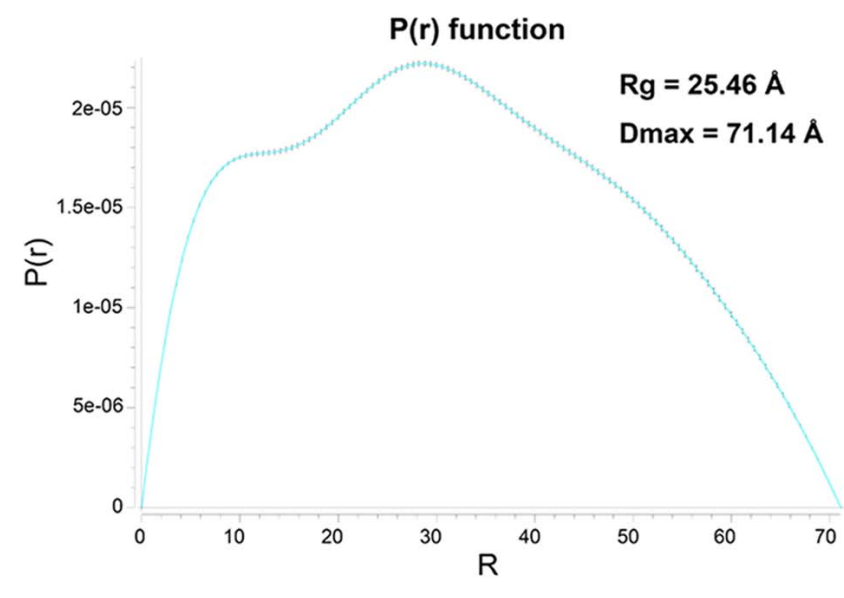

C

\begin{tabular}{|cccc|}
\hline & $\begin{array}{c}\operatorname{Rg}(\AA) \\
(\text { Guinier) }\end{array}$ & $\begin{array}{c}\operatorname{Rg}(\AA) \\
(\text { Gnom })\end{array}$ & $\begin{array}{c}\mathrm{D}_{\max }(\AA) \\
(\text { Gnom })\end{array}$ \\
\hline $\begin{array}{c}\text { NLP7 PB1 } \\
\text { (dimer) }\end{array}$ & $25.45 \pm 0.01$ & $25.46 \pm 0.1$ & $\sim 71.14$ \\
\hline
\end{tabular}

D

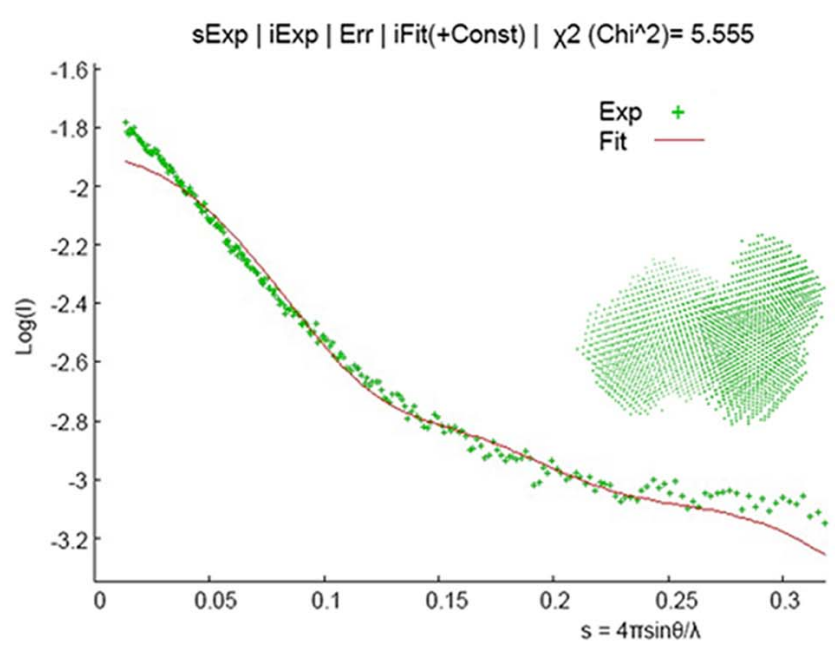

$\mathbf{E}$

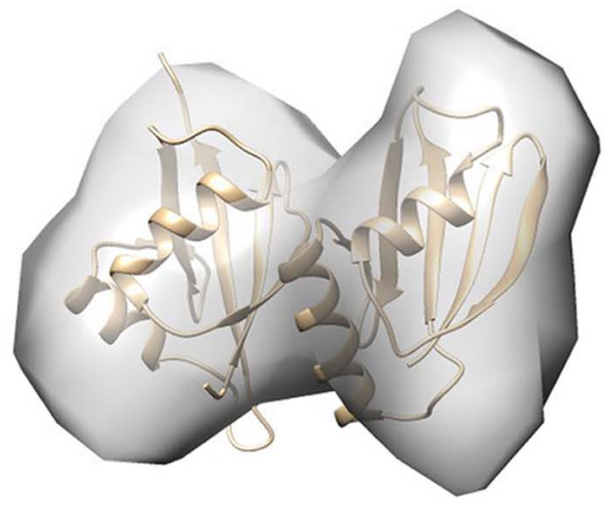

FIGURE 4 | Molecular shape of NLP7 PB1, as determined using small-angle X-ray scattering (SAXS). (A) SAXS curves for the NLP7 PB1 domain at different protein concentrations: $0.9,1.2$, and $1.5 \mathrm{mg} / \mathrm{mL}$. The signal for the $1.5 \mathrm{mg} / \mathrm{mL}$ solution suggests protein aggregation. (B-C) The radius of gyration $\left(\mathrm{R}_{\mathrm{g}}\right)$ and maximum particle size $\left(D_{\max }\right)$ for the NLP7 PB1 domain were estimated based on a Guinier approximation in PRIMUS and the distance distribution function, $\mathrm{P}(\mathrm{r})$, in GNOM. The $P(r)$ function curve suggests that the NLP7 PB1 domain may adopt a dumbbell-like shape. The NLP7 PB1 domain has an average $R_{g}$ of $25.45 \AA$ and a $D_{\text {max }}$ of $71.14 \AA$. (D) Scattering curve (green points, experimental data) for the NLP7 PB1 domain with scattering vector values (q) of $0.007-0.35 \AA$, where $q=4 \pi \sin \theta / \lambda$. The experimental data were fitted with a curve (red) based on the low-resolution dummy atom model. (E) Superimposition of the three-dimensional structure envelope (gray) reconstructed from the dummy atom model in Situs 2.7.3 and the homology model (gold) built in Modeler 9.15 (template: 4MJS). This shows that the NLP7 PB1 domain exists in a dimeric form in solution. 


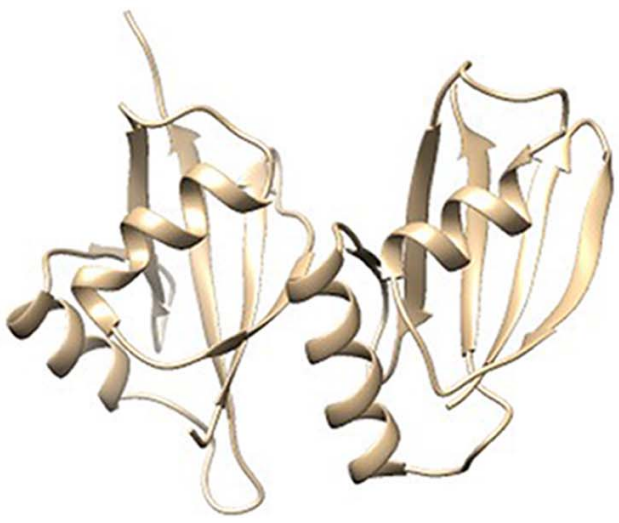

B Monomer A

Interface area

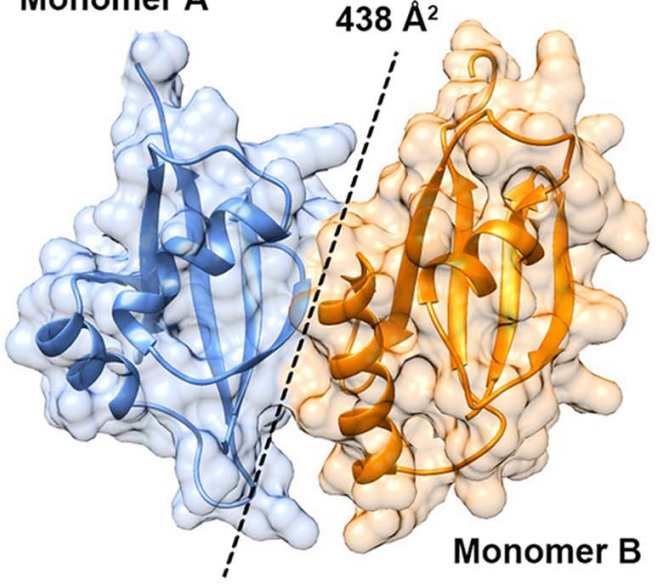

C

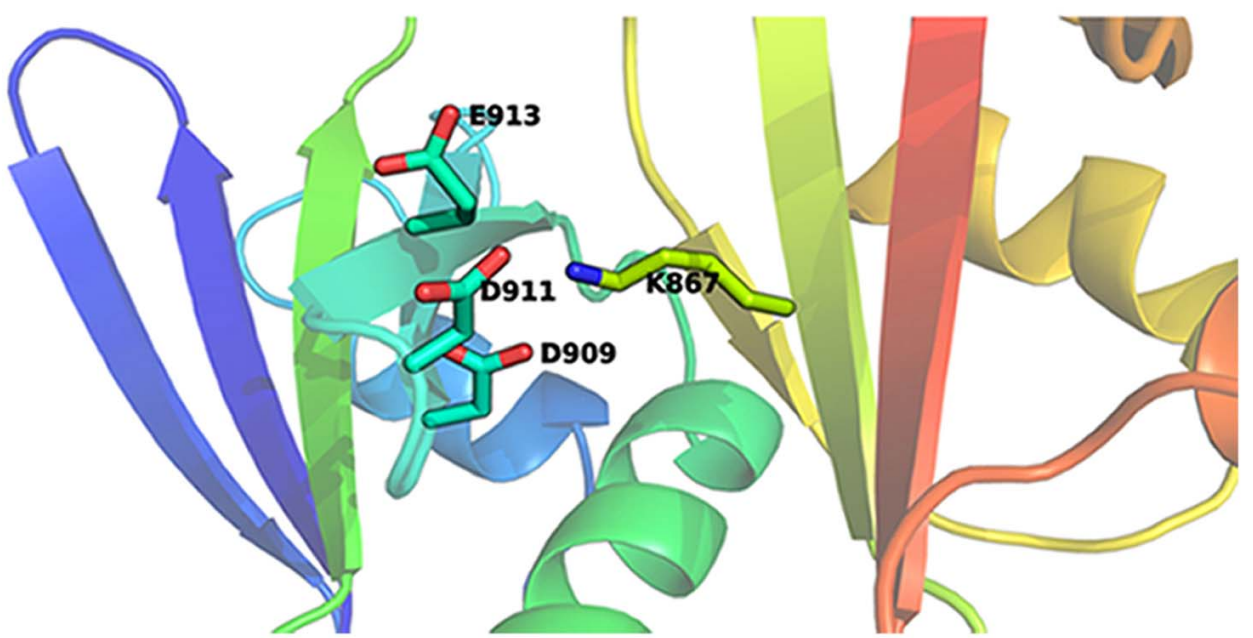

D
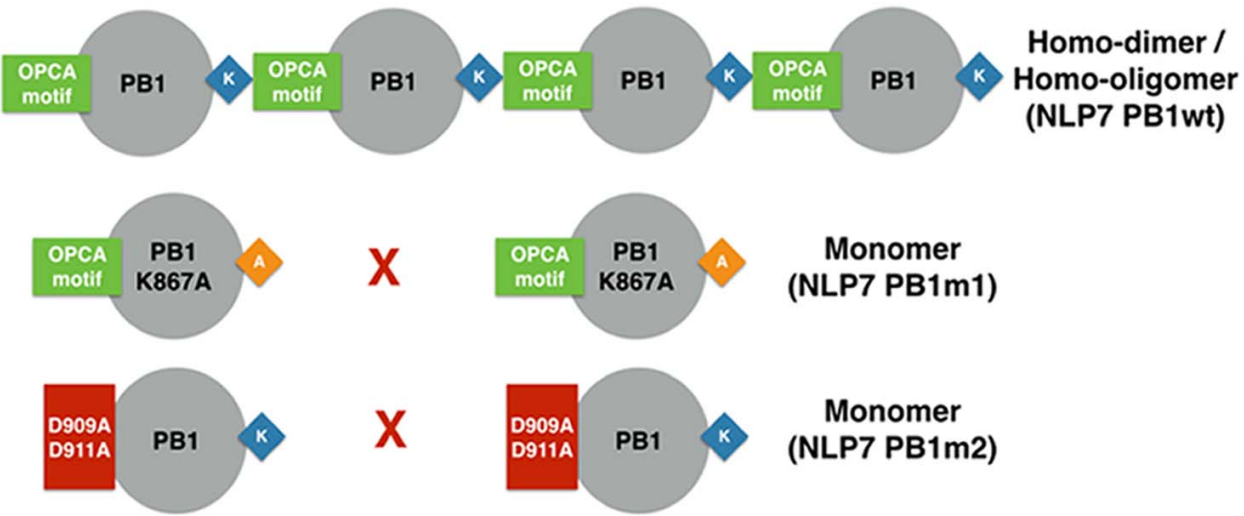

Monomer (NLP7 PB1m2)

FIGURE 5 | Structural model for NLP7 PB1 indicates the residues in the dimeric interface. (A) A structural model of the dimeric NLP7 PB1 domain was built using homology modeling with a structure template (PDB ID: 4MJS). (B) The interface area of the NLP7 PB1 dimer was approximately $438 \AA^{2}$, calculated using the DALI server. Monomer A is colored blue and monomer B is colored orange. (C) From the structural model, the residues K867 (type I motif) and D909/D911/E913/D922 (type II motif) of NLP7 PB1 are involved in salt-bridge interactions to form the homodimer. (D) The proposed model shows that NLP7 PB1 could form homodimers or homo-oligomers via the interactions between the K867 and OPCA motifs. Two mutant lines, NLP7 PB1m1 (with K867A) and NLP7 PB1m2 with (D909A and D911A), were used to confirm the important roles of these motifs in the interaction between two PB1 domains. 
formed a homogeneous (monodispersed) system after advanced purification (Figures 6C-E). Our SEC and DLS results thus provide evidence that the oligomerization/dimerization of the NLP7 PB1 domain is mediated by its two functional motifs.

\section{DISCUSSION}

\section{Presence of Conserved Residues and the OPCA Motif in the PB1 Domain Across Land Plant Lineages Suggests Ancient Origin of Protein-Protein Interaction Function of PB1 Domain in Regulating Nitrate-Signaling Pathway in Land Plants}

In the PB1 domain, there are four key amino-acid residues (K in the type II motif and D, D, and E in the OPCA motif) that are responsible for mediating protein-protein interactions (Sumimoto et al., 2007; Konishi and Yanagisawa, 2019). By aligning PB1 domain residues, we found that these four residues remain almost identical at the amino-acid level across bryophyte and angiosperm species, suggesting an ancient origin of and functional constraint on these four residues (Figure 1). Sitedirected mutagenesis assays have shown that mutations of these four residues can inhibit PB1-PB1 interaction in both humans (Nakamura et al., 1998; Yoshinaga et al., 2003) and Arabidopsis (Konishi and Yanagisawa, 2019).

With extensive sampling of 61 NLP-like sequences from 17 representative species across bryophytes and angiosperms, we reconstructed an across-Viridiplantae NLP phylogeny that suggested the inference of at least four duplication events across the sampled species during the evolutionary history of NLP genes. The monophyly of the NLP homologs obtained from bryophytes and seed plant suggests that these two clades originated in the most recent common ancestor predating the divergence of seed plant (Figure 2, divergence I). Within seed plant, the NLP genes formed three well-supported monophyletic groups: Group 2 (aLRT: 1; BI: -), Group 1 (aLRT: 0.99; BI: 1), and Group 3 (aLRT: 1; BI: 1). Group 2 diverged first, followed by divergence between Group 3 and Group 1 (Figure 2, divergence II and III). Within each group, a general NLP gene divergence pattern was identified. The NLP gene of A. trichopoda (brown box) is the basal lineage, followed by divergence of monocot NLP genes (Oryza and Zea mays, green box), then a basal angiosperm (A. coerulea, sky blue box), and lastly the NLP genes of other angiosperms (purple box). This diversification pattern suggests that the angiosperm NLP genes completed their lineage sorting and diverged prior to angiosperm diversification. We identified three distinct NLP clades, similar to what previous studies have found (Suzuki et al., 2013; Mu and Luo, 2019; Liu and Bisseling, 2020; Wu et al., 2020). Taking the AtNLP genes as an example, AtNLP8 and AtNLP9 belong to Group 2; AtNLP6 and AtNLP7 belong to Group 3, and AtNLP1-5 belong to Group 1, which is consistent with previous studies (Mu and Luo, 2019; Liu and Bisseling, 2020; Wu et al., 2020). Combining these findings with functional studies of NLPs, we postulate that the NLP genes underwent functional divergence following the clade divergence. For example, AtNLP8 is known to be a master regulator of nitrate-promoted seed germination (Yan et al., 2016), AtNLP7 is regarded as a master regulator of nitrate-inducible genes (Konishi and Yanagisawa, 2019), and AtNLP1 may contribute to nodulation in plants not in the nitrogen-fixation clade (NFC) (Wu et al., 2020). The topology published by Liu and Bisseling (2020) suggested an ancient and independent origin of the nodulation and nitrate-fixation functions of NLPs in angiosperms. Contrary to their findings, our topology suggests that the nodulation and nitrate-fixation functions may be recently evolved rather than ancestral (Figure 2 and Supplementary Figure 1).

NIN-like protein genes obtained from selected Viridiplantae species contains two key domains, including RWP-RK and PB1 domain. RWP-RK domain was regarded as a "plant-specific" domain (Mu and Luo, 2019), suggesting RWP-RK domain originating from common ancestor of Viridiplantae. However, RWP-RK domain is identified in oomycete, but PB1 domain absent (Yin et al., 2020). Their finding suggests RWP-RK domain may evolve since the last eukaryotic ancestor. Altogether, we can hypothesize that common ancestor of Viridiplantae gained PB1 domain, forming NLP gene. Following species divergence, NLP gene maintained in few green algae lineages (e.g., M. pusilla) and multiple duplication events occurred after emergence of common ancestor of land plant. The lack of fern $N L P$ genes in this study is due to the absence of complete $N L P$ sequences in the databases we searched. In addition, the key residues for regulating the AtNLP7 PB1 domain interaction appeared to have been conserved in land plants (Figure 1, K867 and D909/D911/E913, red arrows; Figure 5). Therefore, we postulate that these key residues of the PB1 domain evolved prior to land-plant diversification. A possible explanation for why multiple NLP duplicates have been preserved across land plants could be related to the wide range of environmental conditions in land-plant habitats. M. polymorpha occurs in moist environments where nitrates are sufficient. However, when diverse land habitats were colonized, such as savannas and mountains, the uptake of nitrogen may have been more difficult than in moist environments. In addition, the duplicated NLP genes may have provided opportunities for symbiosis with bacteria to form nodules facilitating nitrogen uptake, as with the Group 1 NLP genes of legumes (Huang et al., 2018; Liu et al., 2019).

\section{AtNLP7 PB1 Domain Mediates Self-Oligomerization Through Dimerization}

Arabidopsis thaliana NLP7 is known to be a master regulator of the nitrate-signaling pathway in Arabidopsis (Konishi and Yanagisawa, 2013, 2014, 2019). The PB1 domain is located at the C-terminus in AtNLP7 and has been shown to be responsible for protein-protein interactions in regulating nitrate-inducible gene expression (Konishi and Yanagisawa, 2019). For example, the expression level of NIA1, a nitrate reductase gene, is reduced in AtNLP7 PB1-domain mutant lines (Konishi and Yanagisawa, 2019). In addition, shoot fresh weight in PB1-domain mutant 
A

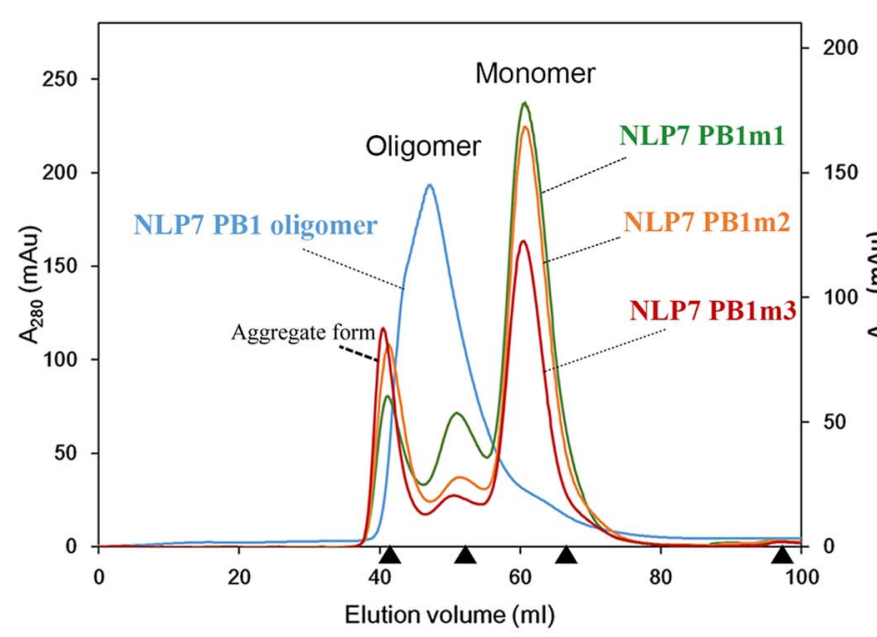

B

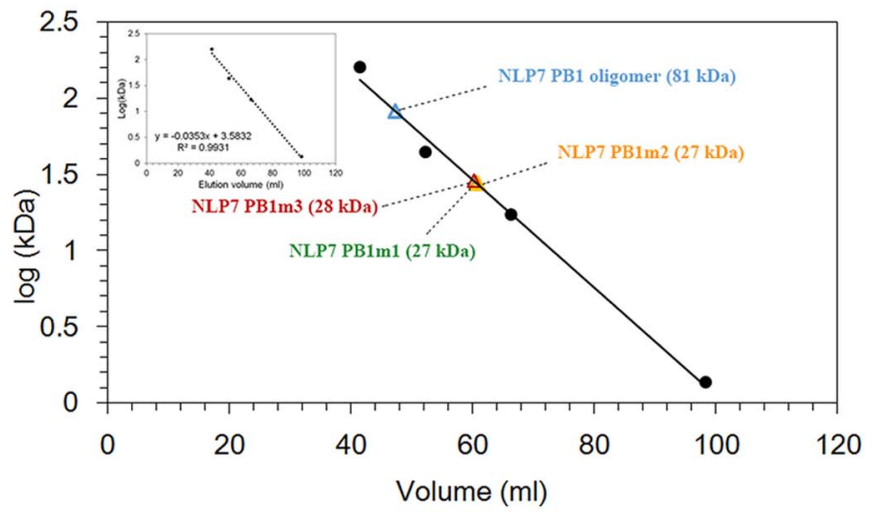

C

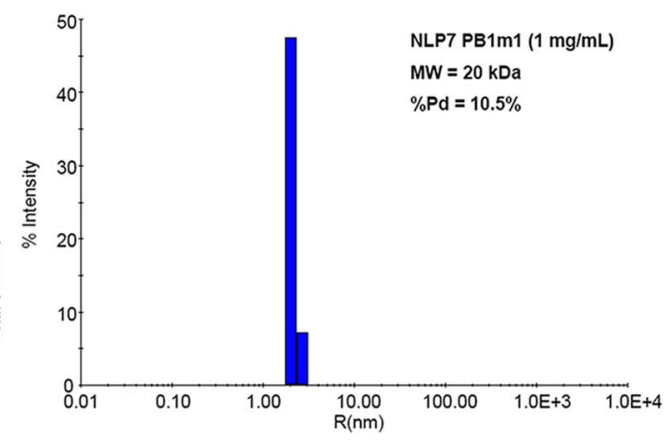

D

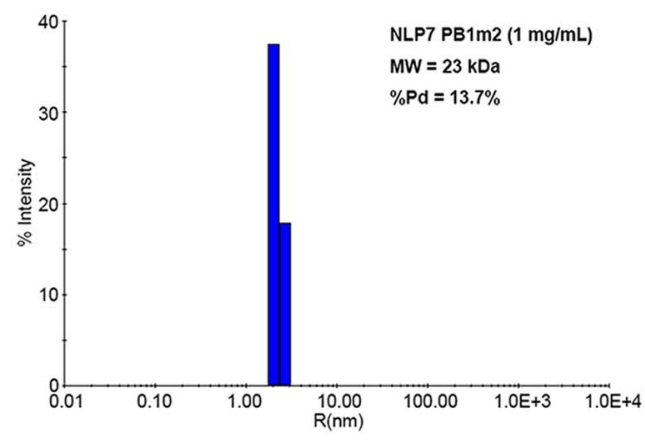

E

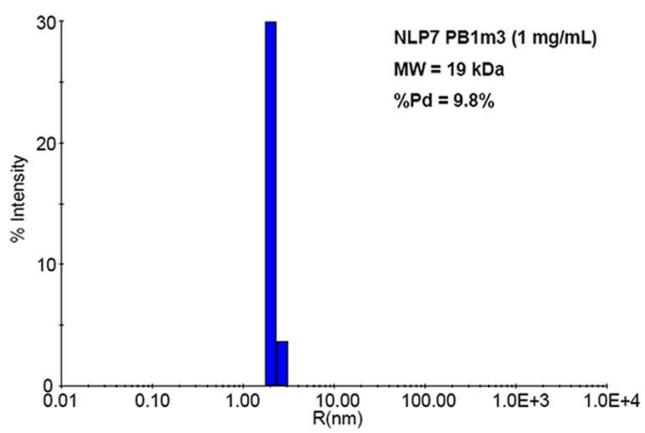

FIGURE 6 | The NLP7 PB1 oligomer is maintained by the interaction between type I and II motifs. (A) Size-exclusion chromatography of NLP7 PB1wt (blue), PB1m1 (K867A, green), PB1m2 (D909A/D911A, orange), and PB1m3 (K867A/D909A/D911A, red) indicated that NLP7 PB1wt forms an oligomer, whereas all mutants were eluted in monomeric form in solution. (B) The molecular weights of NLP7 PB1wt and the mutants were estimated based on the calibration curve for protein standard markers. (C-E) Consistent with panels (A,B), dynamic light scattering showed that NLP7 PB1m1, PB1m2, and PB1m3, in which the hypothetical interactive residues had been mutated, existed as monomers in solution.

lines is lower than that in the wild type (Konishi and Yanagisawa, 2019). Even though the PB1 domain is known to mediate protein-protein interactions, the oligomerization state of this domain remains unknown. Understanding the oligomerization state of a target protein or domain allows us to gain knowledge about how traits or organs developed and differentiated. For example, hetero- and homodimer forms of the MADS box protein have been found to be associated with subtle quantitative differences in stamen shape in maize (Abraham-Juarez et al., 2020). In this study, we used SAXS and structural modeling to confirm that the AtNLP7 PB1 domain could form homodimers via salt-bridge interactions in vitro (Figures 4, 5). In addition, we showed that mutation of the lysine residue (K867) in the type II motif and aspartic acid residues (D909A/D911A) in the OPCA motif would change the oligomerization state of the PB1 domain from homodimer to monomer (Figure 5). Combined with the previous analysis of expression levels of AtNLP7 PB1 mutants conducted by Konishi and Yanagisawa (2019), we hypothesize that if the homodimer form of AtNLP7 PB1 is lost, the growth of Arabidopsis roots would be reduced. Our findings provide insight into how AtNLP is involved, via the PB1 domain at the protein level, in the nitrate-signaling pathway in land plants. 


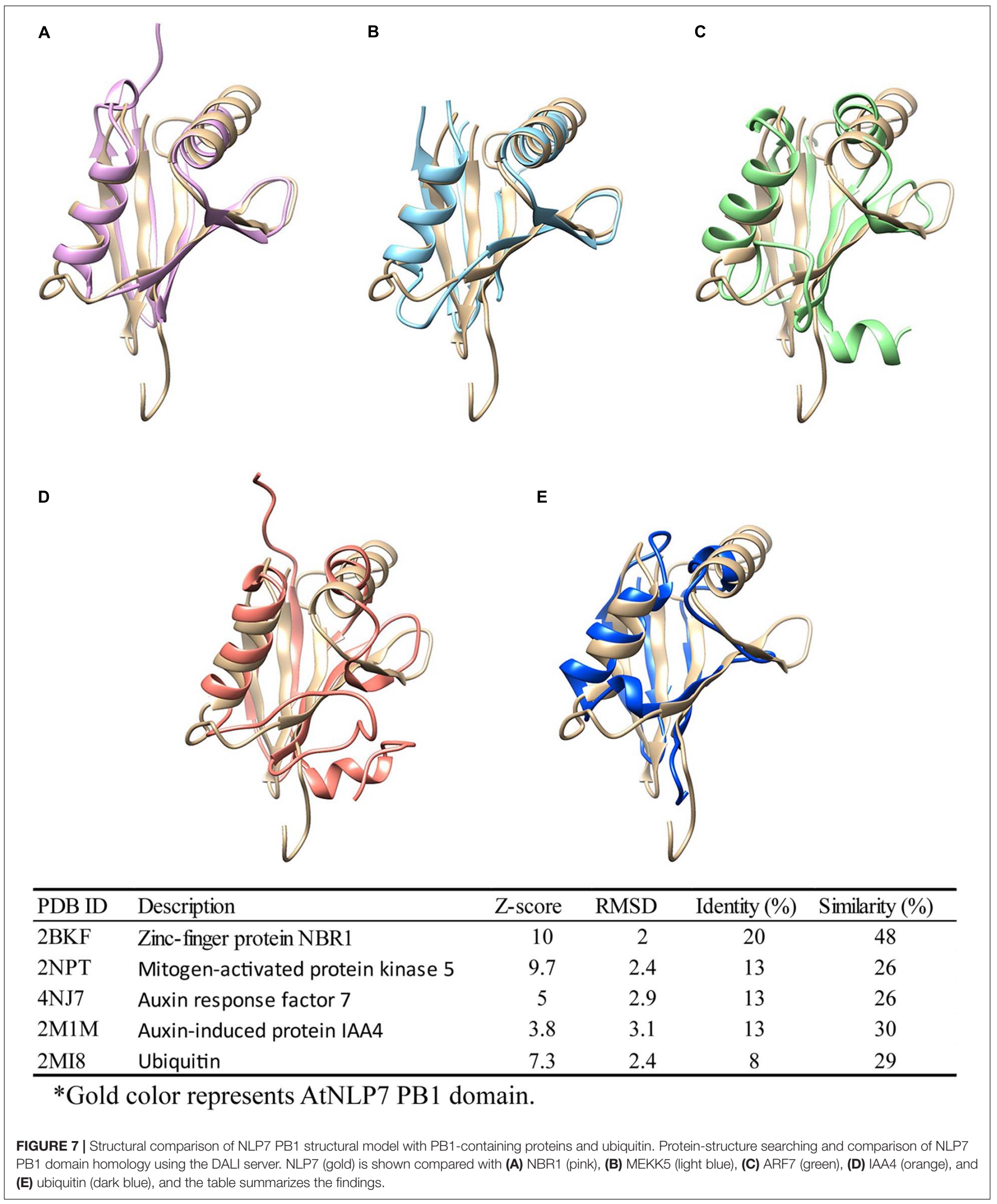




\section{AtNLP7 PB1 Domain Could Also Serve as an Interaction Domain in Mediating the Auxin-Response Regulatory Network in Plants}

As previously reported, the PB1 domain is found in yeast, humans, and plants, and plays diverse roles in different species (Sumimoto et al., 2007). Among these species, PB1 domaincontaining genes play different roles. For example, PB1 domain interaction between PB1 domain-containing $\mathrm{p}^{\text {phox }} \mathrm{p}^{\text {and }} \mathrm{p} 40^{\text {phox }}$ genes is involved in the activation of nicotinamide adenine dinucleotide phosphate (NADPH) oxidase in mammals (Cross and Segal, 2004; Sumimoto et al., 2005). In plants, PB1 domaincontaining genes are involved in nitrogen fixation, nitrate signaling, and seed germination (Yan et al., 2016; Konishi and Yanagisawa, 2019). In earlier functional studies, sequence (Tian and Skolnick, 2003) or structure (Wilson et al., 2000) similarities were used to explore and assume protein functions among similar protein sequences or structures (Rost, 2002; Conant and Wolfe, 2008). Therefore, to explore the potential role of the AtNLP7 PB1 domain, we performed a structural comparison of this domain using the DALI server (Holm, 2020). We found five candidates with structural identity (Figures 7A-E), ranging from 8 to $20 \%$ (amino acid similarity ranging from 26 to $48 \%$ ). Of these, Auxin response factor 7 (ARF7) and Auxin-induced protein (IAA4) attracted our attention first. Interaction between ARF7 and IAA4 was known to occur via their C-terminal domain, called Domain III/IV, which has recently been identified as a type I/II PB1 domain. This interaction facilitates their homo-/hetero/oligomerization via the same mechanism as AtNLP7 PB1 (Korasick et al., 2014). In addition, another interesting candidate is ubiquitin, which is known to be a targeting protein that links to the lysine residue of proteins targeted for intracellular degradation (Tan et al., 2013). A recent study found that PB1 domain-containing protein kinase $\mathrm{C}$ was able to form heterooligomers to prevent biased assemblage of $\mathrm{p} 62$ protein (Ciuffa et al., 2015). Our structural modeling provides potential roles for the PB1 domain for further exploration of its function in plants.

\section{CONCLUSION}

In summary, our phylogeny shows that divergence of angiosperm $N L P$ genes occurred before angiosperm diversification. In addition, a complete lineage sorting of each group and the maintenance of duplicates within each group (except one possible loss in A. coerulea in Group 1) suggests that the NLP genes may be suitable for revealing evolutionary relationships in plants at high taxonomy levels. Furthermore, our biophysical studies and structural model of the AtNLP7 PB1 domain indicate that this domain can form either homodimers or homo-oligomers in regulating the nitrate-response network.

\section{DATA AVAILABILITY STATEMENT}

The datasets presented in this study can be found in online repositories. The names of the repository/repositories and accession number(s) can be found in the article/Supplementary Material.

\section{AUTHOR CONTRIBUTIONS}

K-TH, T-JY, and Y-SC: conceptualization. K-TH, T-JY, and Y-HL: data curation. K-TH and T-JY: formal analysis and writing-original draft. Y-SC: funding acquisition and supervision. K-TH, T-JY, Y-HL, and Y-SC: methodology. K-TH, Y-HL, and Y-SC: writing-review and editing. All authors contributed to the article and approved the submitted version.

\section{FUNDING}

Financial support was provided to Y-SC by the Taiwan Ministry of Science and Technology (MOST 109-2311-B-002-026 and MOST 109-2311-B-002-012).

\section{ACKNOWLEDGMENTS}

We thank Yi-Fang Tsay for discussing and for providing the pDL2Nx-NLP7 PB1 plasmid. Portions of this research were carried out at beamline (BL23A1) at the NSRRC (Hsinchu, Taiwan). We are grateful to the staff of the Technology Commons, College of Life Science at National Taiwan University, for help with use of the spectrophotometer, luminescent image analyzer, and structural biology core. We would also like to thank Anthony Abram for editing and proofreading this manuscript.

\section{SUPPLEMENTARY MATERIAL}

The Supplementary Material for this article can be found online at: https://www.frontiersin.org/articles/10.3389/fpls.2021. 672035/full\#supplementary-material

Supplementary Figure 1 | Phylogram of NLP genes.

Supplementary Figure 2 | SDS-PAGE of purified 6xHis-tagged NLP7 PB1 domain. SDS-PAGE reveals that the molecular weight of the purified 6xHis-tagged NLP7 PB1wt domain is $15.6 \mathrm{kDa}$.

Supplementary Figure 3 | SDS-PAGE of purified 6xHis-tagged NLP7 PB1m1 domain. SDS-PAGE reveals that the molecular weight of the purified 6 xHis-tagged NLP7 PB1m1 domain is $15.6 \mathrm{kDa}$.

Supplementary Figure 4 | SDS-PAGE of purified 6xHis-tagged NLP7 PB1m2 domain. SDS-PAGE reveals that the molecular weight of the purified $6 x$ His-tagged NLP7 PB1m2 domain is $15.6 \mathrm{kDa}$.

Supplementary Table 1 | List of species and sequences used in phylogeny reconstruction.

Supplementary Table 2 | Primers used in the construction of the NLP7 PB1 domain and mutants.

Supplementary Table 3 | Pairwise nucleotide similarity among AtNLP7 PB1 and identified PB1 domain containg homologs.

Supplementary Data 1 | Alignment of NLP genes used in this study. 


\section{REFERENCES}

Abraham-Juarez, M. J., Schrager-Lavelle, A., Man, J., Whipple, C., Handakumbura, P., Babbitt, C., et al. (2020). Evolutionary variation in MADS box dimerization affects floral development and protein abundance in maize. Plant Cell 32, 3408-3424. doi: $10.1105 /$ tpc.20.00300

Anisimova, M., and Gascuel, O. (2006). Approximate likelihood-ratio test for branches: a fast, accurate, and powerful alternative. Syst. Biol. 55, 539-552. doi: 10.1080/10635150600755453

Anisimova, M., Gil, M., Dufayard, J. F., Dessimoz, C., and Gascuel, O. (2011). Survey of branch support methods demonstrates accuracy, power, and robustness of fast likelihood-based approximation schemes. Syst. Biol. 60, 685-699. doi: 10.1093/sysbio/syr041

Castaings, L., Camargo, A., Pocholle, D., Gaudon, V., Texier, Y., Boutet-Mercey, S., et al. (2009). The nodule inception-like protein 7 modulates nitrate sensing and metabolism in Arabidopsis. Plant J. 57, 426-435. doi: 10.1111/j.1365-313x. 2008.03695.x

Chardin, C., Girin, T., Roudier, F., Meyer, C., and Krapp, A. (2014). The plant RWP-RK transcription factors: key regulators of nitrogen responses and of gametophyte development. J. Exp. Bot. 65, 5577-5587. doi: 10.1093/jxb/eru261

Ciuffa, R., Lamark, T., Tarafder, A. K., Guesdon, A., Rybina, S., Hagen, W. J., et al. (2015). The selective autophagy receptor $\mathrm{p} 62$ forms a flexible filamentous helical scaffold. Cell Rep. 11, 748-758. doi: 10.1016/j.celrep.2015.03.062

Conant, G. C., and Wolfe, K. H. (2008). Turning a hobby into a job: how duplicated genes find new functions. Nat. Rev. Genet. 9, 938-950. doi: 10.1038/nrg2482

Cross, A. R., and Segal, A. W. (2004). The NADPH oxidase of professional phagocytes-prototype of the NOX electron transport chain systems. Biochim. Biophys. Acta 1657, 1-22. doi: 10.1016/j.bbabio.2004.03.008

Darriba, D., Taboada, G., Doallo, R., and Posada, D. (2012). jModelTest 2: more models, new heuristics and parallel computing. Nat. Methods 9:772. doi: 10. 1038/nmeth.2109

Edgar, R. C. (2004). MUSCLE: multiple sequence alignment with high accuracy and high throughput. Nucleic. Acids. Res. 32, 1792-1797. doi: 10.1093/nar/gkh340

Eswar, N., Webb, B., Marti-Renom, M. A., Madhusudhan, M. S., Eramian, D., Shen, M. Y., et al. (2006). Comparative protein structure modeling using modeller. Curr. Protoc. Bioinform. 50, 2-9. doi: 10.1002/0471250953.bi0506s15

Finet, C., Timme, R. E., Delwiche, C. F., and Marlétaz, F. (2010). Multigene phylogeny of the green lineage reveals the origin and diversification of land plants. Curr. Biol. 20, 2217-2222. doi: 10.1016/j.cub.2010.11.035

Franke, D., and Svergun, D. I. (2009). DAMMIF, a program for rapid ab-initio shape determination in small-angle scattering. J. Appl. Crystallogr. 42, 342-346. doi: $10.1107 / \mathrm{s} 0021889809000338$

Goodstein, D. M., Shu, S., Howson, R., Neupane, R., Hayes, R. D., Fazo, J., et al. (2012). Phytozome: a comparative platform for green plant genomics. Nucleic Acids Res. 40, D1178-D1186.

Gowri, G., Kenis, J. D., Ingemarsson, B., Redinbaugh, M. G., and Campbell, W. H. (1992). Nitrate reductase transcript is expressed in the primary response of maize to environmental nitrate. Plant Mol. Biol. 18, 55-64. doi: 10.1007/ bf00018456

Guindon, S., Dufayard J. F., Lefort V., Anisimova, M., Hordijk, W., and Gascuel, O. (2010). New algorithms and methods to estimate maximum-likelihood phylogenies: assessing the performance of PhyML 3.0. Syst. Biol. 59, 307-321. doi: $10.1093 /$ sysbio/syq010

Hall, T. A. (1999). BioEdit: a user-friendly biological sequence alignment editor and analysis program for windows 95/98/NT. Nucleic. Acids. Symp. Ser. 41, 95-98. doi: 10.14601/Phytopathol_Mediterr-14998u1.29

Holm, L. (2020). DALI and the persistence of protein shape. Protein Sci. 29, 128-140. doi: 10.1002/pro.3749

Huang, C. T., Hsin, K. T., Wang, C. N., Liu, C. T., and Kao, W. Y. (2018) Phylogenetic analyses of Bradyrhizobium symbionts associated with invasive Crotalaria zanzibarica and its coexisting legumes in Taiwan. Syst Appl. Microbiol. 41:6.

Kikhney, A. G., and Svergun, D. I. (2015). A practical guide to small angle X-ray scattering (SAXS) of flexible and intrinsically disordered proteins. FEBS Lett. 589, 2570-2577. doi: 10.1016/j.febslet.2015.08.027

Konarev, P. V., Volkov, V. V., Sokolova, A. V., Koch, M. H. J., and Svergun, D. I. (2003). PRIMUS: a windows PC-based system for small-angle scattering data analysis. J. Appl. Cryst. 36, 1277-1282. doi: 10.1107/s0021889803012779
Konishi, M., and Yanagisawa, S. (2010). Identification of a nitrate-responsive ciselement in the Arabidopsis NIR1 promoter defines the presence of multiple cis-regulatory elements for nitrogen response. Plant J. 63, 269-282. doi: 10. 1111/j.1365-313x.2010.04239.x

Konishi, M., and Yanagisawa, S. (2013). Arabidopsis NIN-like transcription factors have a central role in nitrate signalling. Nat. Commun. 4:1617.

Konishi, M., and Yanagisawa, S. (2014). Emergence of a new step towards understanding the molecular mechanisms underlying nitrate-regulated gene expression. J. Exp. Bot. 65, 5589-5600. doi: 10.1093/jxb/eru267

Konishi, M., and Yanagisawa, S. (2019). The role of protein-protein interactions mediated by the PB1 domain of NLP transcription factors in nitrate-inducible gene expression. BMC Plant Biol. 19:90. doi: 10.1186/s12870-019-1692-3

Korasick, D. A., Westfall, C. S., Lee, S. G., Nanao, M. H., Dumas, R., Hagen, G., et al. (2014). Molecular basis for AUXIN RESPONSE FACTOR protein interaction and the control of auxin response repression. Proc. Natl. Acad. Sci. U S A. 111, 5427-5432. doi: 10.1073/pnas.1400074111

Krissinel, E., and Henrick, K. (2007). Inference of macromolecular assemblies from crystalline state. J. Mol. Biol. 372, 774-797. doi: 10.1016/j.jmb.2007.05.022

Liu, J., and Bisseling, T. (2020). Evolution of NIN and NIN-like genes in relation to nodule symbiosis. Genes (Basel) 11:777. doi: 10.3390/genes11070777

Liu, J., Rutten, L., Limpens, E., van der Molen, T., van Velzen, R., Chen, R., et al. (2019). A remote cis-regulatory region is required for NIN expression in the pericycle to initiate nodule primordium formation in Medicago truncatula. Plant Cell 31, 68-83. doi: 10.1105/tpc. 18.00478

Liu, K. H., Niu, Y., Konishi, M., Wu, Y., Du, H., Sun Chung, H., et al. (2017). Discovery of nitrate-CPK-NLP signaling in central nutrient-growth networks. Nature 545, 311-316. doi: 10.1038/nature22077

Marchive, C., Roudier, F., Castaings, L., Brehaut, V., Blondet, E., Colot, V., et al. (2013). Nuclear retention of the transcription factor NLP7 orchestrates the early response to nitrate in plants. Nat. Commun. 4:1713.

$\mathrm{Mu}, \mathrm{X}$., and Luo, J. (2019). Evolutionary analyses of NIN-like proteins in plants and their roles in nitrate signaling. Cell Mol. Life. Sci 76, 3753-3764. doi: 10.1007/s00018-019-03164-8

Nakamura, R., Sumimoto, H., Mizuki, K., Hata, K., Ago, T., Kitajima, S., et al. (1998). The PC motif: a novel and evolutionarily conserved sequence involved in interaction between p40phox and p67phox, SH3 domain-containing cytosolic factors of the phagocyte NADPH oxidase. Eur. J. Biochem. 251, 583-589. doi: 10.1046/j.1432-1327.1998.2510583.x

Pettersen, E. F., Goddard, T. D., Huang, C. C., Couch, G. S., Greenblatt, D. M., Meng, E. C., et al. (2004). UCSF Chimera-a visualization system for exploratory research and analysis. J. Comput. Chem. 25, 1605-1612. doi: 10.1002/jcc.20084

Rost, B. (2002). Enzyme function less conserved than anticipated. J Mol Biol. 318, 595-608. doi: 10.1016/s0022-2836(02)00016-5

Sato, T., Maekawa, S., Konishi, M., Yoshioka, N., Sasaki, Y., Maeda, H., et al. (2016). Direct transcriptional activation of BT genes by NLP transcription factors is a key component of the nitrate response in Arabidopsis. Biochem. Biophys. Res. Commun. 483, 380-386. doi: 10.1016/j.bbrc.2016.12.135

Schauser, L., Wieloch, W., and Stougaard, J. (2005). Evolution of NIN-like proteins in Arabidopsis, rice, and Lotus japonicus. J. Mol. Evol. 60, 229-237. doi: 10.1007/ s00239-004-0144-2

Schwarz, G. (1978). Estimating the dimension of a Model. Ann. Stat. 6, 461-464. doi: $10.1214 /$ aos/1176344136

Sumimoto, H., Kamakura, S., and Ito, T. (2007). Structure and function of the PB1 domain, a protein interaction module conserved in animals, fungi, amoebas, and plants. Sci. STKE 28:re6. doi: 10.1126/stke.4012007re6

Sumimoto, H., Miyano, K., and Takeya, R. (2005). Molecular composition and regulation of the Nox family $\mathrm{NAD}(\mathrm{P}) \mathrm{H}$ oxidases. Biochem. Biophys. Res. Commun. 338, 677-686. doi: 10.1016/j.bbrc.2005.08.210

Suzuki, W., Konishi, M., and Yanagisawa, S. (2013). The evolutionary events necessary for the emergence of symbiotic nitrogen fixation in legumes may involve a loss of nitrate responsiveness of the NIN transcription factor. Plant Signal. Behav. 8:e25975. doi: 10.4161/psb.25975

Svergun, D. (1992). Determination of the regularization parameter in indirecttransform methods using perceptual criteria. J. Appl. Crystallogr. 25, 495-503. doi: $10.1107 / \mathrm{s} 0021889892001663$

Tamura, K., Stecher, G., Peterson, D., Filipski, A., and Kumar, S. (2013). MEGA6: molecular evolutionary genetics analysis version 6.0. Mol. Biol. Evol. 30, 2725 2729. doi: $10.1093 / \mathrm{molbev} / \mathrm{mst} 197$ 
Tan, W. H., Gilmore, E. C., and Baris, H. N. (2013). "Human developmental genetics," in Emery and Rimoin's Principles and Practice of Medical Genetics, eds D. L. Rimoin, R. E. Pyeritz, and B. R. Korf (Philadelphia, PA: Elsevier Science).

Tian, W., and Skolnick, J. (2003). How well is enzyme function conserved as a function of pairwise sequence identity? J. Mol. Biol. 333, 863-882. doi: 10.1016/ j.jmb.2003.08.057

Walker, R. L., Burns, I. G., and Moorby, J. (2001). Responses of plant growth rate to nitrogen supply: a comparison of relative addition and $\mathrm{N}$ interruption treatments. J. Exp. Bot. 52, 309-317. doi: 10.1093/jxb/52.355.309

Wang, R., Xing, X., Wang, Y., Tran, A., and Crawford, N. M. (2009). A genetic screen for nitrate regulatory mutants captures the nitrate transporter gene NRT1.1. Plant Physiol. 151, 472-478. doi: 10.1104/pp.109.14 0434

Wilson, C. A., Kreychman, J., and Gerstein, M. (2000). Assessing annotation transfer for genomics: quantifying the relations between protein sequence, structure and function through traditional and probabilistic scores. J. Mol. Biol. 297, 233-249. doi: 10.1006/jmbi.2000.3550

Wriggers, W., and Chacón, P. (2001). Using Situs for the registration of protein structures with low-resolution bead models from X-ray solution scattering. J. Appl. Cryst. 34, 773-776. doi: 10.1107/S00218898010 12869

Wu, Z., Liu, H., Huang, W., Yi, L., Qin, E., Yang, T., et al. (2020). GenomeWide identification, characterization, and regulation of RWP-RK gene family in the nitrogen-fixing clade. Plants (Basel) 11:1178. doi: 10.3390/plants90 91178

Yan, D., Easwaran, V., Chau, V., Okamoto, M., Ierullo, M., Kimura, M., et al. (2016). NIN-like protein 8 is a master regulator of nitrate-promoted seed germination in Arabidopsis. Nat. Commun. 7:13179.

Yin, M., Zhang, Z., Xuan, M., Feng, H., Ye, W., Zheng, X., et al. (2020). Conserved subgroups of the plant-specific RWP-RK transcription factor family are present in oomycete pathogens. Front. Microbiol. 11:1724. doi: 10.3389/fmicb.2020. 01724

Yoshinaga, S., Kohjima, M., Ogura, K., Yokochi, M., Takeya, R., Ito, T., et al. (2003). The PB1 domain and the PC motif-containing region are structurally similar protein binding modules. EMBO J. 22, 4888-4897. doi: 10.1093/emboj/cdg475

Conflict of Interest: The authors declare that the research was conducted in the absence of any commercial or financial relationships that could be construed as a potential conflict of interest.

Copyright (c) 2021 Hsin, Yang, Lee and Cheng. This is an open-access article distributed under the terms of the Creative Commons Attribution License (CC BY). The use, distribution or reproduction in other forums is permitted, provided the original author(s) and the copyright owner(s) are credited and that the original publication in this journal is cited, in accordance with accepted academic practice. No use, distribution or reproduction is permitted which does not comply with these terms. 